قسم الأحياء الدقيقة - كلية الطب البيطرى جامعة البعث - سوريا

\title{
COMPARATIVE LABORATORY STUDY ON THE EFFECTS OF CERTAIN TYPES OF LIPIDS ON THE CONCENTRATION OF PLASMA TOTAL CHOLESTEROL (TC) AND HIGH DENSITY LIPOPROTEIN (HDL-C) IN HAMSTER
} (With 3 Tables and 3 Figures)

By

\section{ALHELAL; A. ALAWAD and N. HAPRA}

(Received at 19/8/2010)

دراسة مخبريّة مقارنة لتأثيرات بعض أنواع الدهون على منسوب الكولسترول الكلي

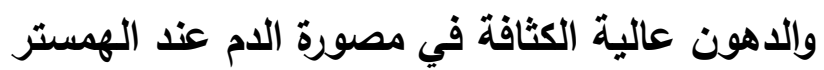

\section{محد الهلال ، عواد العواد * ، ناجح هبرة}

* قسم وظائف الأعضاء - كلية الطب البيطرى - جامعة البعث

أجريت التجربة على /128/ ذكر من الهمستر السليمة صحيّاً ، حيث قسّمت الحيوانات إلى أربع

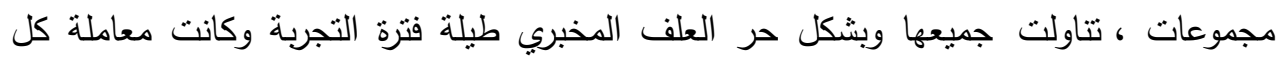
مجموعة كالتالي: المجموعة الضابطة: تتاولت العلف المخبري المحافظ على البقاء فقط. مجموعة ولثة

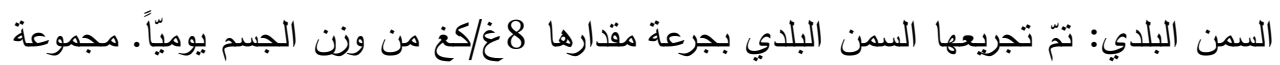
زيت الزيتون: نّّ تجريعها زيت الزيتون بجرعة مقدارها 8 غ/كغ من وزن الجسم يومياً. مجموعة زيت

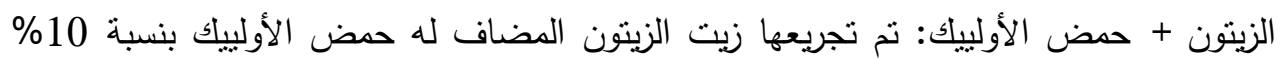

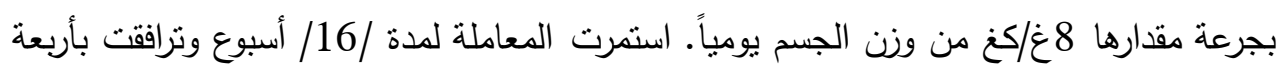

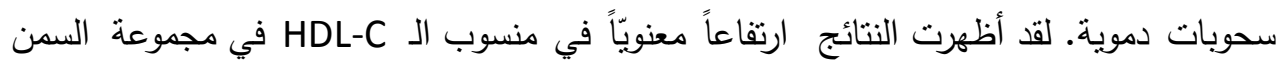

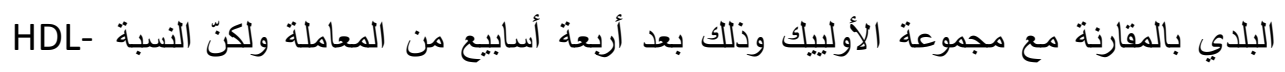
كانت مرتفعة معنويًاً بققدار 28\% C/TC

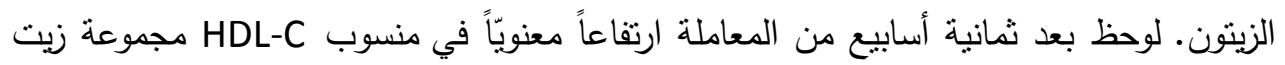




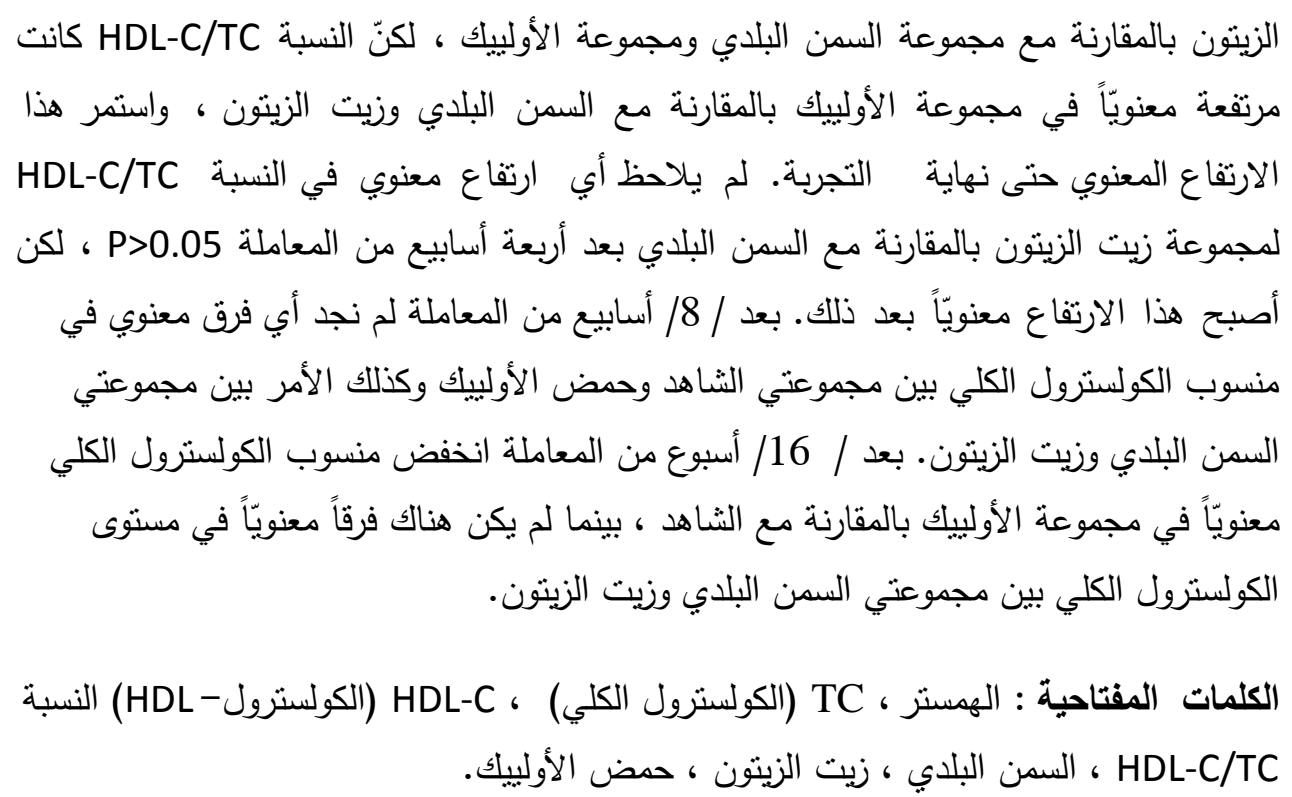

\section{SUMMARY}

The experiment was applied on /128/ healthy male hamsters divided into four groups. Each group had laboratorial diet, freely, during the period of the experiment: Domestic Ghee Group were dosed domestic ghee $8 \mathrm{~g} / \mathrm{kg}$ of the body weight daily; Olive Oil Group were dosed olive oil $8 \mathrm{~g} / \mathrm{kg}$ of the body weight; Olive Oil+ Oleic Acid Group were dosed olive oil supplemented by $10 \%$ of oleic acid $8 \mathrm{~g} / \mathrm{kg}$ of the body weight daily and Control Group which had laboratorial diet that made maintenance ration. Four blood samples were collected during the experiment for 16 weeks. The results showed a significant increase $(\mathrm{P}=0.0002)$ of the HDL-C concentration in the domestic ghee group in comparison with the group treated with oleic acid in four weeks of treatment, but HDL-C/TC ratio was significantly increased $(\mathrm{P}=0.0000)$ approximately $28 \%$ for the group treated with oleic acid in comparison with the groups of domestic ghee and olive oil. There was a significant increase in concentration of HDL-C olive oil group in comparison with the group treated with oleic acid and domestic ghee group in /8/ weeks, but HDL-C/TC ratio was significantly increased $(\mathrm{P}=0.0000)$ in the group treated with oleic acid in comparison with the groups of domestic ghee and olive oil. This significant increase remained in $/ 12 /$ and $/ 16 /$ weeks of treatment. There was no significant increase $(\mathrm{P}>0.05)$ in HDL-C/TC ratio of olive oil group in comparison with domestic ghee group in /4/ weeks of treatment, but this increase became 
significant $(\mathrm{P}=0.0000)$ after that. There was no significant difference $(\mathrm{P}>0.05)$ in TC concentration between the control and oleic acid groups and also domestic ghee and olive oil groups $(\mathrm{P}>0.05)$ in $/ 8 /$ weeks of treatment. At /16/ weeks of treatment, TC concentration of oleic acid group decreased significantly $(\mathrm{P}=0.0000)$ in comparison with control group, while there was no significant difference $(\mathrm{P}>0.05)$ between the domestic ghee and olive oil groups.

Key words: Hamster, (HDL-C) HDL-Cholesterol, (TC) Total Cholesterol, HDL-C/TC, domestic ghee, olive oil, oleic acid.

\section{INTRODUCTION}

\section{المقدمـة}

تعد الثحميات إحدى المركبات العضوية الأساسية في الجسم الحي وذللك لدورها

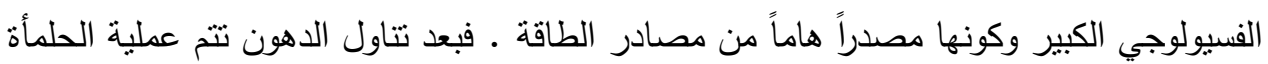

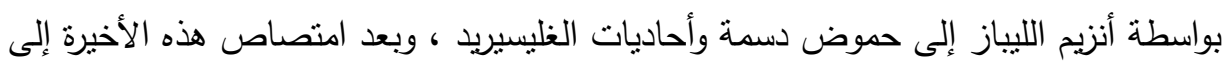

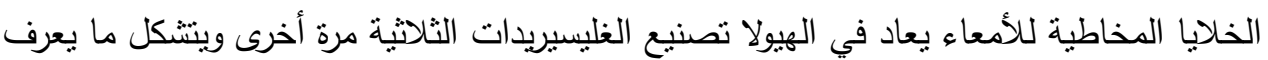

باسم الدقائق الكيلوسية ( Chylomicrons) ، وهي عبارة عن قطيرات دهنية مجهرية كروية تتشكل أساساً من ثلاثثات الغليسيريد مع جزء صغير من الثحميات الفسفورية وأسترات الكولسترول

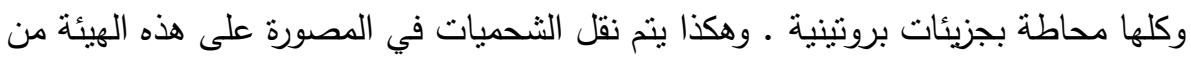

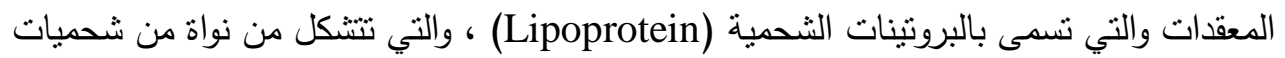

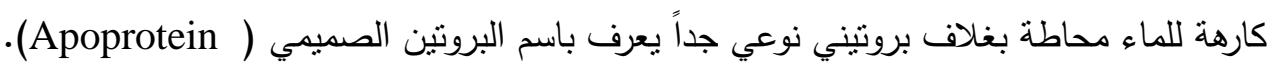

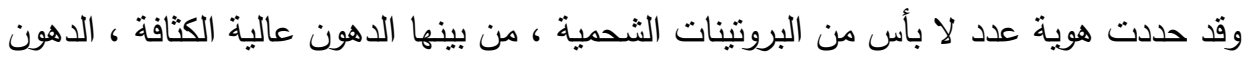

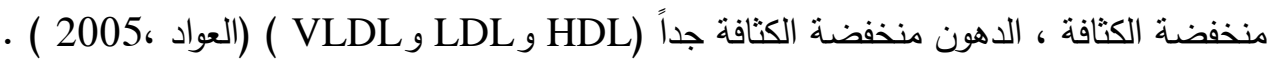
يقوم الـ HDL بنقل الكولسترول من مختلف الأعضاء إلى الكبد ليستقلب ويطرح خارج الجسم

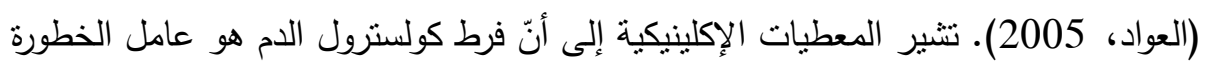

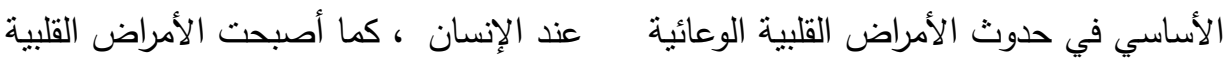
الوعائية المسبب رقم واحد للموت عند الإنسان في الولايات المتحدة الأمريكية منذ عام 1990

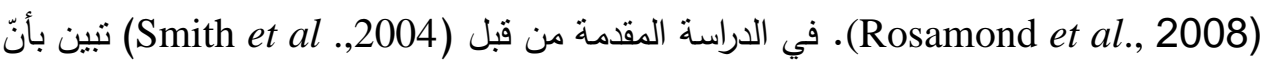

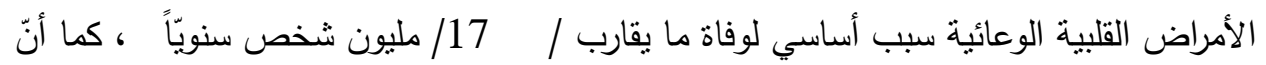

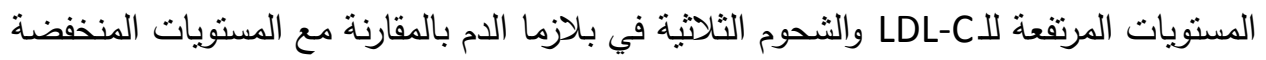


مثرابطة مع زيادة خطورة أمراض القلب التاجية. تعتبر الأغذية الغنية بالأحماض الدسمة

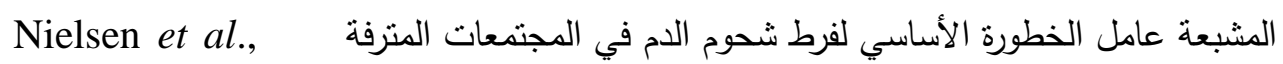

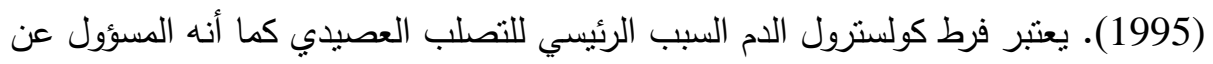

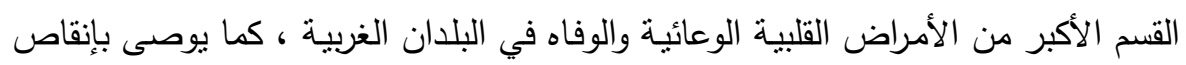
مستوى الأحماض الدسمة المشبعة في الغذاء في هذه المجتمعات (Carleton et al., 1991). White et al., 1997; Bennett et al., 1995; Sessions and Salter, 1994) بيّن (Salter et al., 1998;

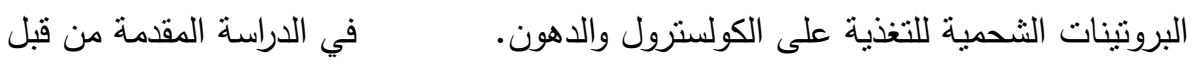
(Mangiapane et al., 1999)

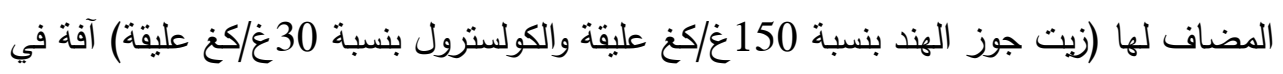
الأبهر الصاعد والقوس الأبهرية وذلك بعد أربعة أسابيع من التخذية ، وازداد تطور الآفة عندما

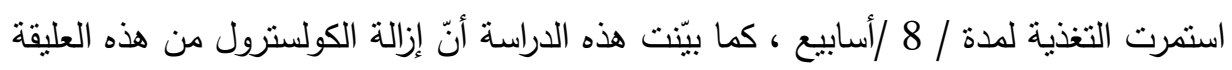
أوقف تطور الآفة. لقد أثبت (Perona et al., 2003 (انخفاض الكولسترول الكلي عند الإنسان

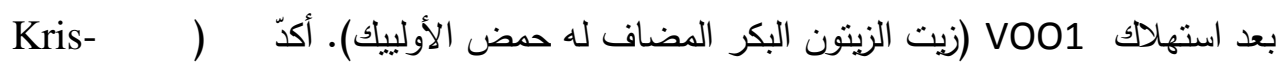

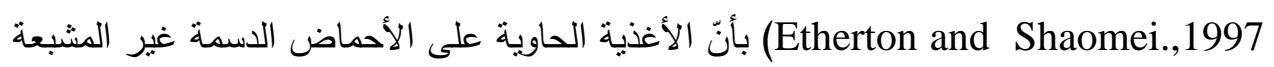

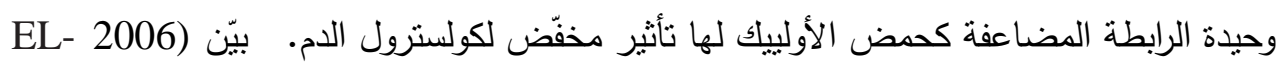
(beshbishy et al .,

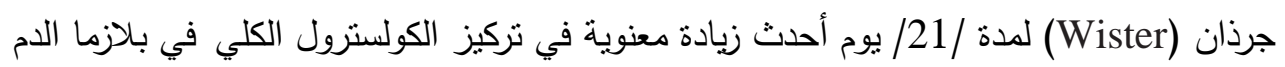

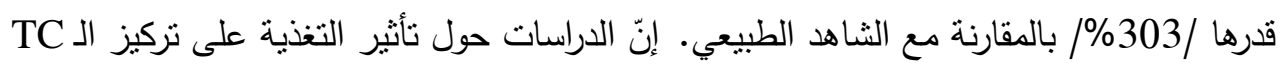

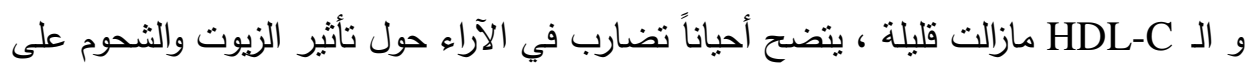

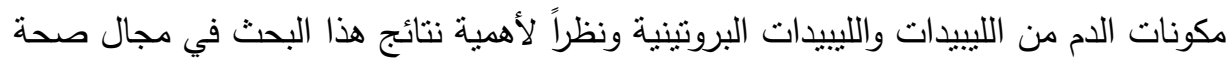

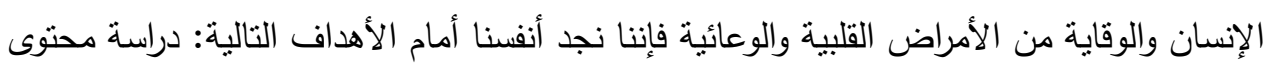

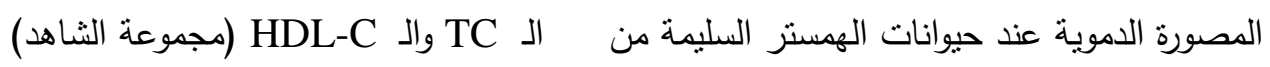
وكذلك دراسة محتواها عند حيوانات الهمستر السليمة من المع يارين السابقين بعد تتاول زيت الهن الزيتون والسمن البلدي وكذلك زيت الزيتون المضاف له حمض الأولييك بنسبة 10\%

\section{MATERIALS and METHODS مواد وطر ائق البحث}


حيوانات التجرية والتغذية: شملت الدراسة /128/ همتراً جميعها كانت من الذكور السليمة

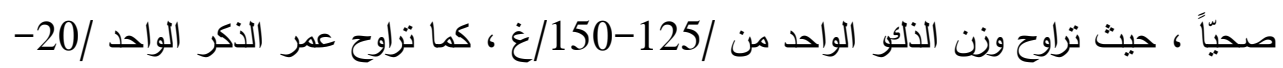

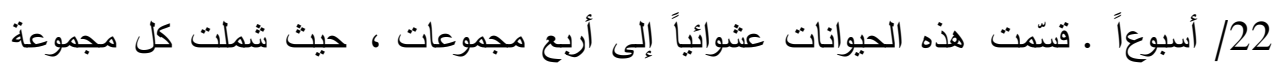

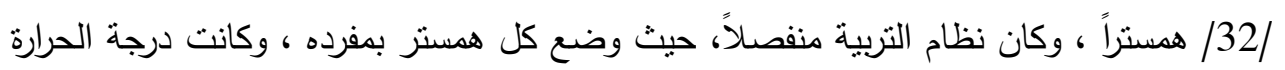

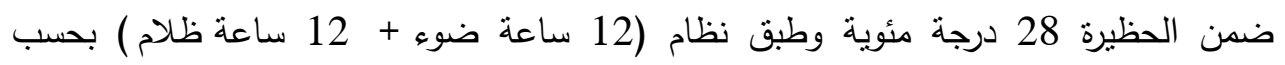

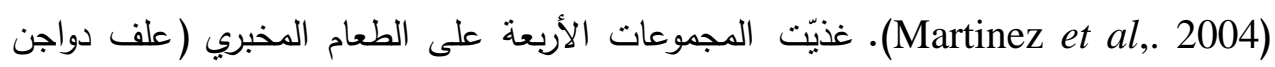

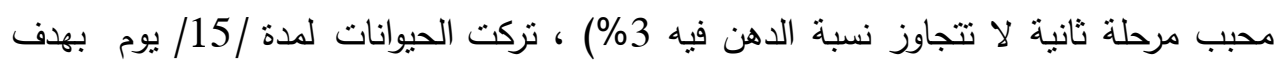

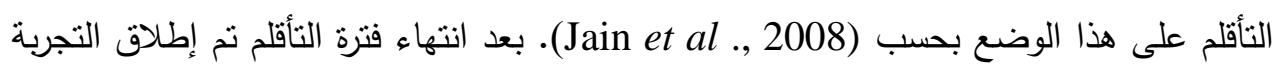

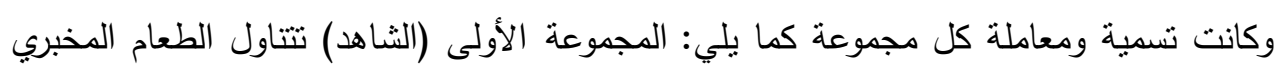
فقط وبشكل حر، في حين تتاولت المجموعة الثانية (مجموعة السمن البلدي) الطعام المخبري

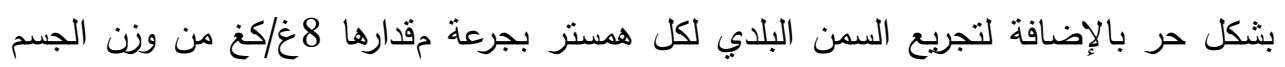

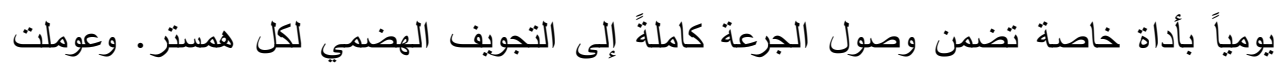

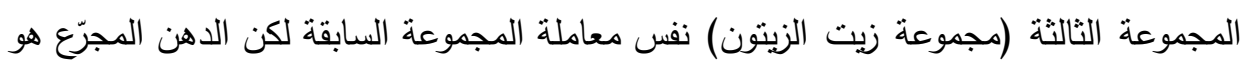

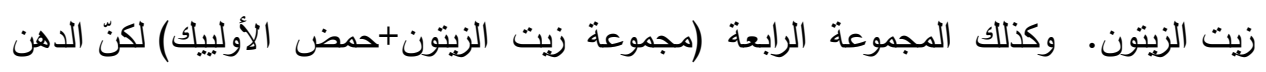
المجرّع هو زيت الزيتون المضاف له حمض الأولييك بنسبة 10\%.

\section{جمع عينات الام والقياسات الدموية}

جمع العينات الدموية: تم سحب الدم عن طريق تقب الجيب الخلف الحجاجي بواسطة أنابيب شعرية مطلية بمانع تخثر (الهيبارين) بحسب (Wilson et al., 2006) وذلك بعد تصويم

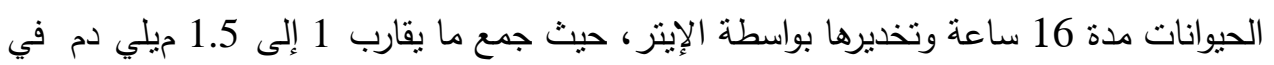
كل مرة ، يبلغ حجم الدم الدائر بحدود (65- 80 مليلتر دم/كغ من وزن الجسم) (

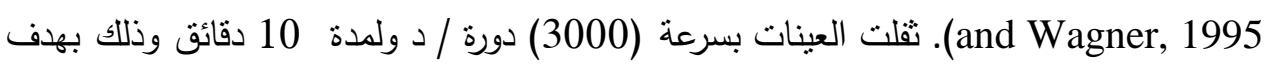

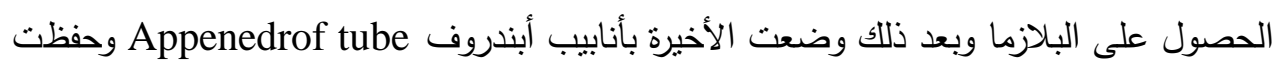

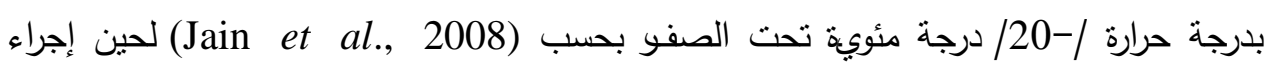
الاختبارات.

القياسات الدموية: لقد أنجزت الاختبارات الكيمياحيوية على عينات البلازما وذلك باستخدام جهاز 20GENESYS (Spectrophotometer) 
الكولسترول الكلي بالطرق الإنزيمية باستخدام الذهر الكيميائية المعيارية ذات الرقم ( 11505)

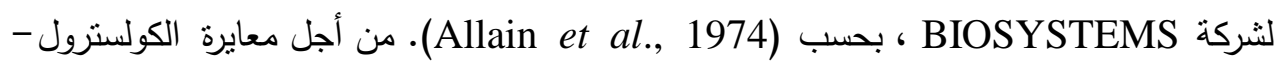

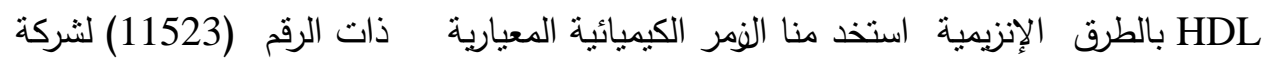

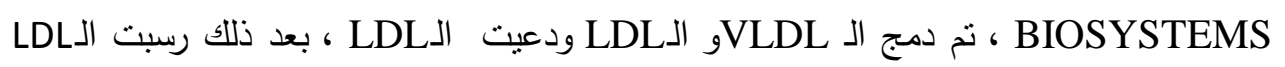
باستخدام: (phosphotungstate and magnesium ions) بحسب ( Weingand and (Daggy 1990 ومن ثم حسب تركيز الـHDL في الطافي. التحليل الإحصائي: تم حساب قيمة P Pذ وذلك في كل مجموعة معاملة وفي كل مرحلة من مراحل التجربة الأربعة بطريقة تحليل التباين وحيد الاتجاه (One-Way ANOVA).

\section{RESULTS}

\section{النتائ-ج}

لقد أظهرت حيوانات الهمستر بشكل عام تحمّلاً جيّداً للتجريع الفموي اليومي للاهون

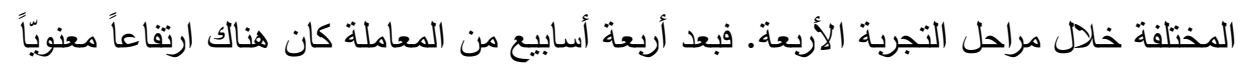

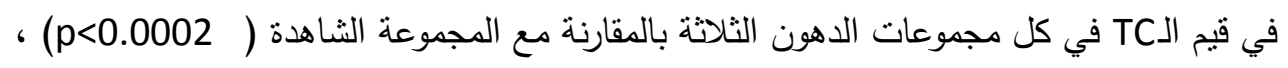
لكنّ الـ TC لمجمعة الأولييك كان الأقل ارتفاعاً بالمقارنة مع الثاهد (

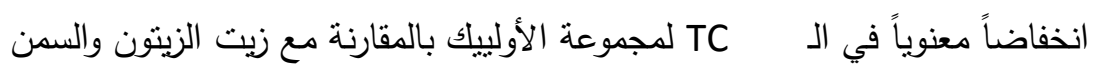

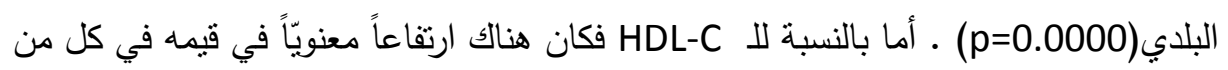
مجموعات الدهون الثلاثة بالمقارنة مع الثاهد (

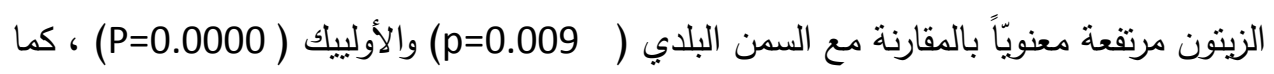
ارتفعت قيمته في السمن البلدي معنويًاً بالمقارنة الأولييك (

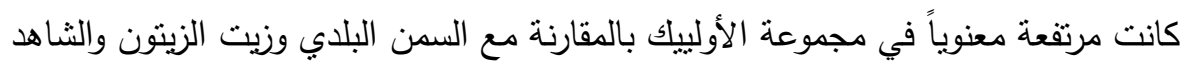

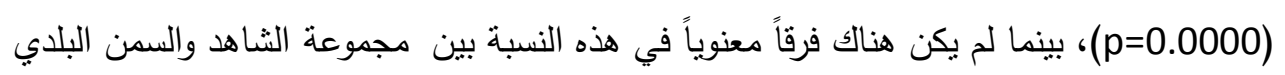

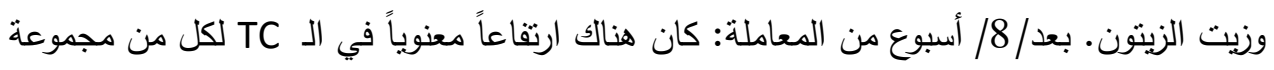

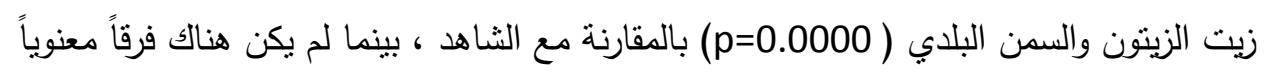

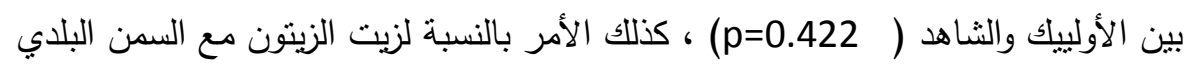

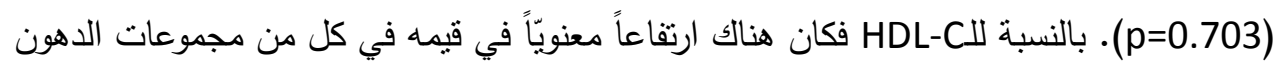

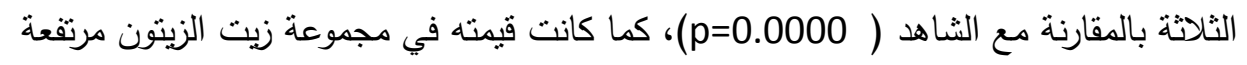

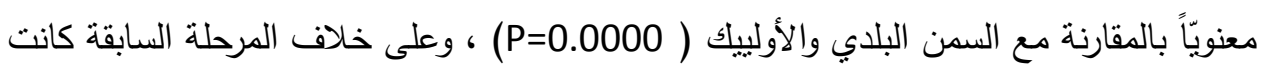




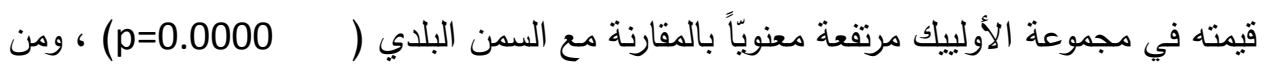
الجدير ذكره أن قيمة الـHDL-C في كل من مجموعتي زيت الزيتون والأولييك مرتفعة بشكل معنوي

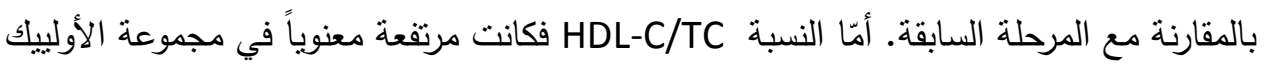

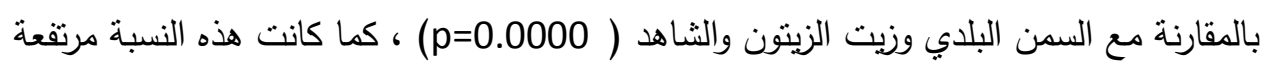

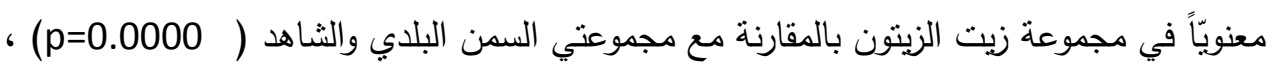

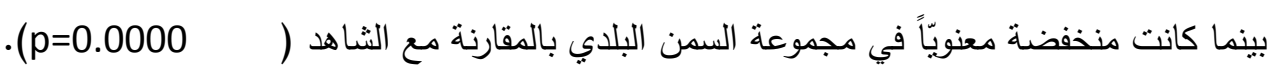

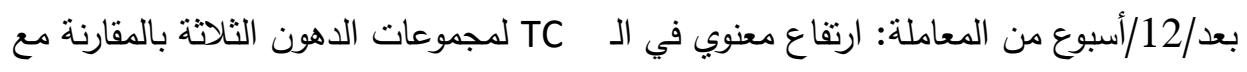

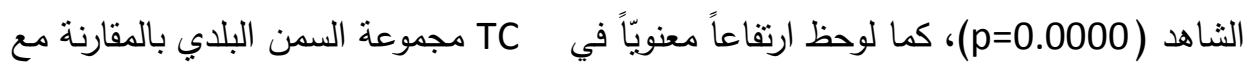

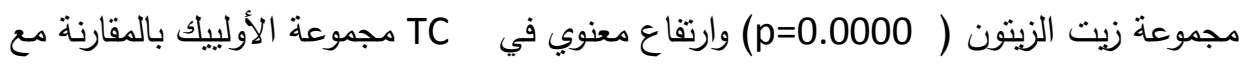

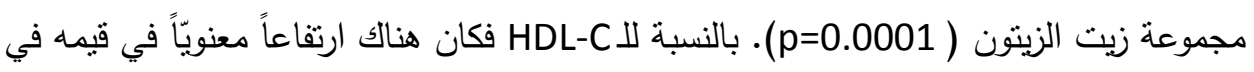

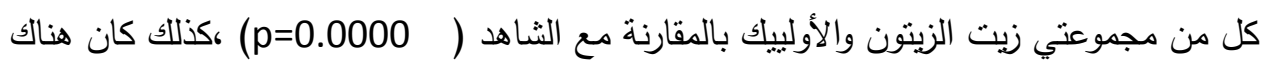

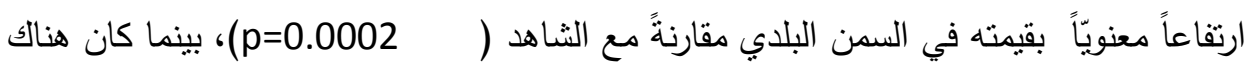
انخفاضاً معنويًاً بقيمته في مجموعة السمن البلدي بالمقارنة مع زيت الزيت الزيتون والأوليكيك

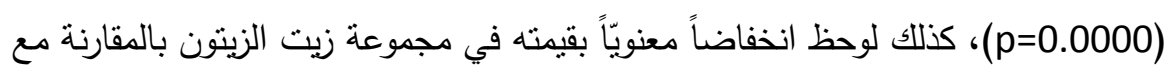

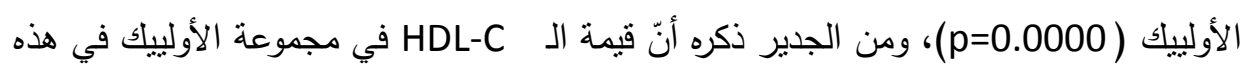

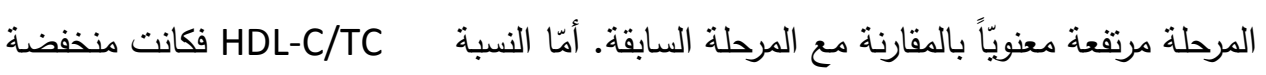

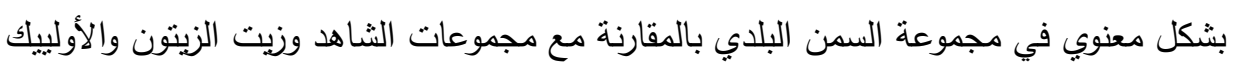

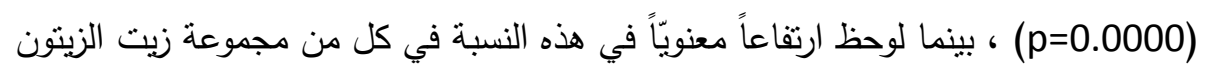

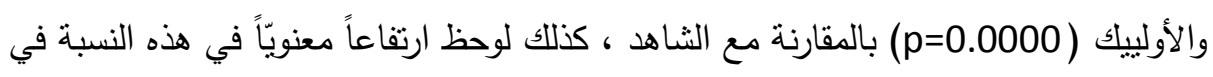

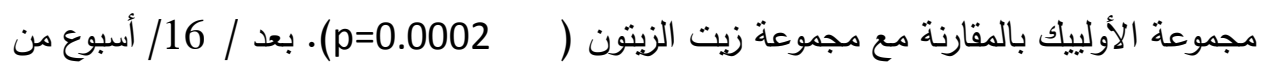

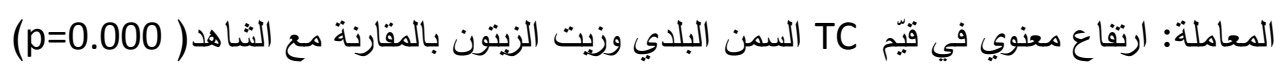

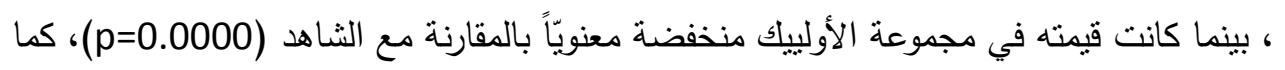

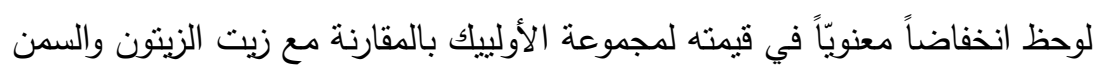

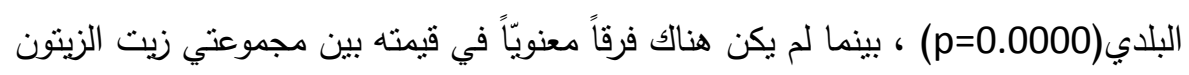

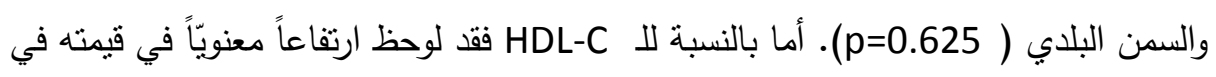

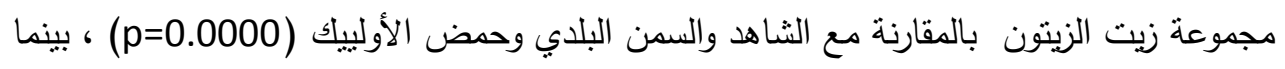

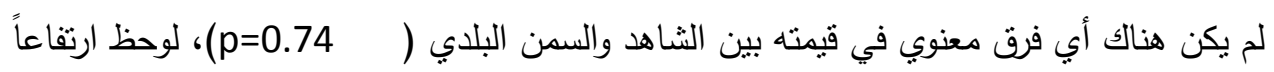

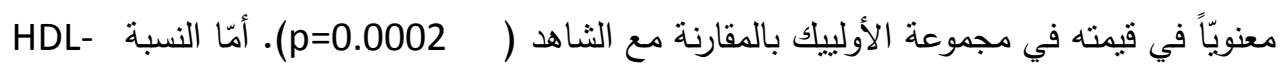


C/TC

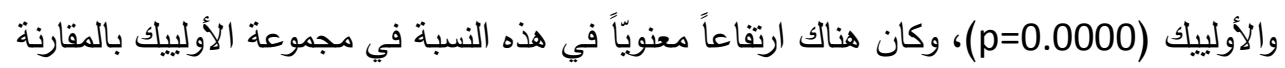

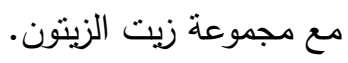

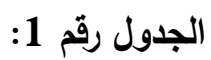

تأثثر تتاول السمن البلدي وزيت الزيتون وزيت الزيبتون المضاف له حمض الأولييك بنسبة 10\% على تركيز الـ(TC) الكولسترول الكلي في بلازما الرم عند الهمستر خلال مراحل التجربة الأربعة.

\begin{tabular}{|c|c|c|c|c|}
\hline $\begin{array}{c}\text { TC } \\
\text { ملغ/16/ أسبوع } 16 \text { / أسبو }\end{array}$ & $\begin{array}{c}\text { TC } \\
\text { ملغ/دل أسبوع } 12 \text { / أسبو }\end{array}$ & $\begin{array}{c}\text { TC } \\
\text { ملغ/دل أسبوع } 1 \text { / } 1 \text { ا }\end{array}$ & $\begin{array}{c}\text { TC } \\
\text { ملغ/دل أسبوع } 14 \text { / السبو }\end{array}$ & المجمو عة \\
\hline $\begin{array}{c}\mathrm{a} \\
59.71 \pm 1.18\end{array}$ & $\begin{array}{c}\mathrm{a} \\
51.16 \pm 2.96\end{array}$ & $\begin{array}{c}\mathrm{a} \\
54.5 \pm 3.26\end{array}$ & $\begin{array}{c}\mathrm{a} \\
39.43 \pm 1.97\end{array}$ & الثشاهد \\
\hline $\begin{array}{c}\mathrm{b} \\
76.62 \pm 3.89\end{array}$ & $\begin{array}{c}\mathrm{b} \\
82.71 \pm 3.73\end{array}$ & $\begin{array}{c}\mathrm{B} \\
74.99 \pm 3.25\end{array}$ & $\begin{array}{c}\mathrm{b} \\
67.53 \pm 1.08\end{array}$ & السمن البلاي \\
\hline $\begin{array}{c}\mathrm{b} \\
75.63 \pm 3.99\end{array}$ & $\begin{array}{c}\mathrm{c} \\
71.48 \pm 1.50\end{array}$ & $\begin{array}{c}\mathrm{B} \\
75.56 \pm 2.61\end{array}$ & $\begin{array}{c}\mathrm{c} \\
72.09 \pm 2.78\end{array}$ & زيت الزيتون \\
\hline $\begin{array}{c}c \\
54.14 \pm 2.27\end{array}$ & $\begin{array}{c}\mathrm{d} \\
77.67 \pm 2.87\end{array}$ & $\begin{array}{c}\mathrm{A} \\
55.77 \pm 2.87\end{array}$ & $\begin{array}{c}\mathrm{d} \\
46.08 \pm 2.68\end{array}$ & زيض الزيتون+ \\
\hline
\end{tabular}

تشير الاختلافات في الأحرف الإنكليزية إلى وجود فروقات معنوية حيث 0.05>p وذلك ضمن

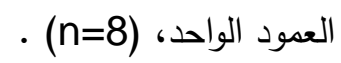

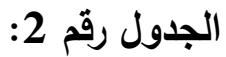

تأثثر تتاول السمن البلدي وزيت الزينون وزيت الزيبنون المضاف له حمض الأولييك بنسبة 10\%

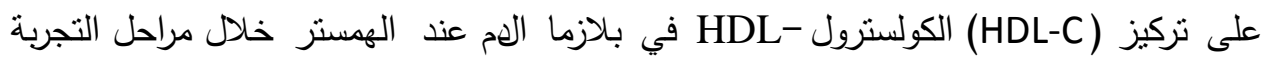
الأربعة.

\begin{tabular}{|c|c|c|c|c|}
\hline $\begin{array}{l}\text { HDL-C } \\
\text { ملغ/دل/16/ أسبوع } \\
\text { / / }\end{array}$ & $\begin{array}{l}\text { HDL-C } \\
\text { ملغ/12/ أسبوع ع / / } 12\end{array}$ & $\begin{array}{l}\text { HDL-C } \\
\text { ملغ/دل أسبوع } \\
\text { / أسبو }\end{array}$ & $\begin{array}{l}\text { HDL-C } \\
\text { ملغ/دل/ أسبوع }\end{array}$ & المجمو عة \\
\hline
\end{tabular}




\begin{tabular}{|c|c|c|c|c|}
\hline $\begin{array}{c}\mathrm{a} \\
43.38 \pm 1.08\end{array}$ & $\begin{array}{c}\mathrm{a} \\
36.41 \pm 1.98\end{array}$ & $\begin{array}{c}\mathrm{A} \\
32.98 \pm 1.51\end{array}$ & $\begin{array}{c}\mathrm{a} \\
23.94 \pm 1.86\end{array}$ & الثشاهد \\
\hline $\begin{array}{c}\mathrm{a} \\
43.00 \pm 2.99\end{array}$ & $\begin{array}{c}\mathrm{b} \\
41.29 \pm 1.81\end{array}$ & $\begin{array}{c}\mathrm{B} \\
37.51 \pm 1.38\end{array}$ & $\begin{array}{c}\mathrm{b} \\
39.30 \pm 1.41\end{array}$ & السمن البلدي \\
\hline $\begin{array}{c}\mathrm{b} \\
61.92 \pm 1.86\end{array}$ & $\begin{array}{c}c \\
55.95 \pm 0.95\end{array}$ & $\begin{array}{c}\mathrm{C} \\
55.43 \pm 2.03\end{array}$ & $\begin{array}{c}\mathrm{c} \\
42.77 \pm 2.91\end{array}$ & زيت الزيتون \\
\hline $\begin{array}{c}c \\
46.97 \pm 1.66\end{array}$ & $\begin{array}{c}\mathrm{d} \\
64.59 \pm 2.54\end{array}$ & $\begin{array}{c}\mathrm{d} \\
46.83 \pm 1.76\end{array}$ & $\begin{array}{c}\mathrm{d} \\
34.99 \pm 2.06\end{array}$ & حضيت الزيتون+ \\
\hline
\end{tabular}

تثير الاختلافات في الأحرف الإنكليزية إلى وجود فروقات معنوية حيث 0.05>p وذلك ضمن

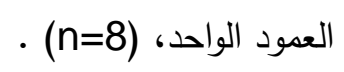

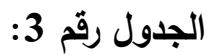

تأثثر تتاول السمن البلدي وزيت الزيتون وزيت الزيبتون المضاف له حمض الأولييك بنسبة 10\% على النسبة HDL-C/TC في بلازما الإم عند الهمستر خلال مراحل التجربة الأربعة.

\begin{tabular}{|c|c|c|c|c|}
\hline $\begin{array}{l}\text { HDL-C/TC } \\
\text { / أسبوع/16/ }\end{array}$ & $\begin{array}{l}\text { HDL-C/TC } \\
\text { /12/ أسبوع / }\end{array}$ & $\begin{array}{c}\text { /8/ HDL-C/TC } \\
\text { أسبوع }\end{array}$ & $\begin{array}{c}\text { /4/ HDL-C/TC } \\
\text { أسبوع }\end{array}$ & المجمو عة \\
\hline $\begin{array}{c}\mathrm{a} \\
0.72 \pm 0.02\end{array}$ & $\begin{array}{c}\mathrm{a} \\
0.71 \pm 0.03\end{array}$ & $\begin{array}{c}\mathrm{a} \\
0.6 \pm 0.02\end{array}$ & $\begin{array}{c}\mathrm{a} \\
0.6 \pm 0.05\end{array}$ & الثشاهد \\
\hline $\begin{array}{c}\mathrm{b} \\
0.56 \pm 0.02\end{array}$ & $\begin{array}{c}\mathrm{b} \\
0.5 \pm 0.02\end{array}$ & $\begin{array}{c}\mathrm{b} \\
0.5 \pm 0.02\end{array}$ & $\begin{array}{c}\mathrm{a} \\
0.58 \pm 0.02\end{array}$ & السمن البلاي \\
\hline $\begin{array}{c}c \\
0.81 \pm 0.02\end{array}$ & $\begin{array}{c}c \\
0.78 \pm 0.02\end{array}$ & $\begin{array}{c}c \mathrm{c} \\
0.73 \pm 0.03\end{array}$ & $\begin{array}{c}\mathrm{a} \\
0.59 \pm 0.03\end{array}$ & زيت الزينون \\
\hline $\begin{array}{c}\mathrm{d} \\
0.86 \pm 0.02\end{array}$ & $\begin{array}{c}\mathrm{d} \\
0.83 \pm 0.01\end{array}$ & $\begin{array}{c}\mathrm{d} \\
0.84 \pm 0.01\end{array}$ & $\begin{array}{c}\mathrm{b} \\
0.76 \pm 0.04\end{array}$ & زميت الزيتون+ \\
\hline
\end{tabular}

تشير الاختلافات في الأحرف الإنكليزية إلى وجود فروقات معنوية حيث 0.05> وذللك ضمن

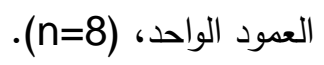

الثكل رقم (1): تركيز الـTC مقدراً بالملغ/دل في بلازما الدم عند الهمستر في كل من مجموعات

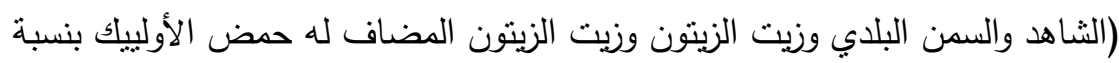

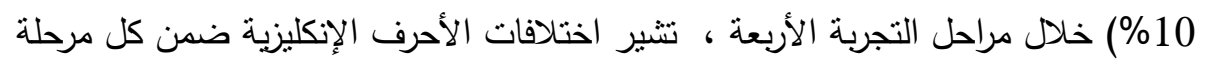
تغذية إلى وجود الفروق المعنوية، حيث أنّ (0.05>p ) ) ، (n=8). 


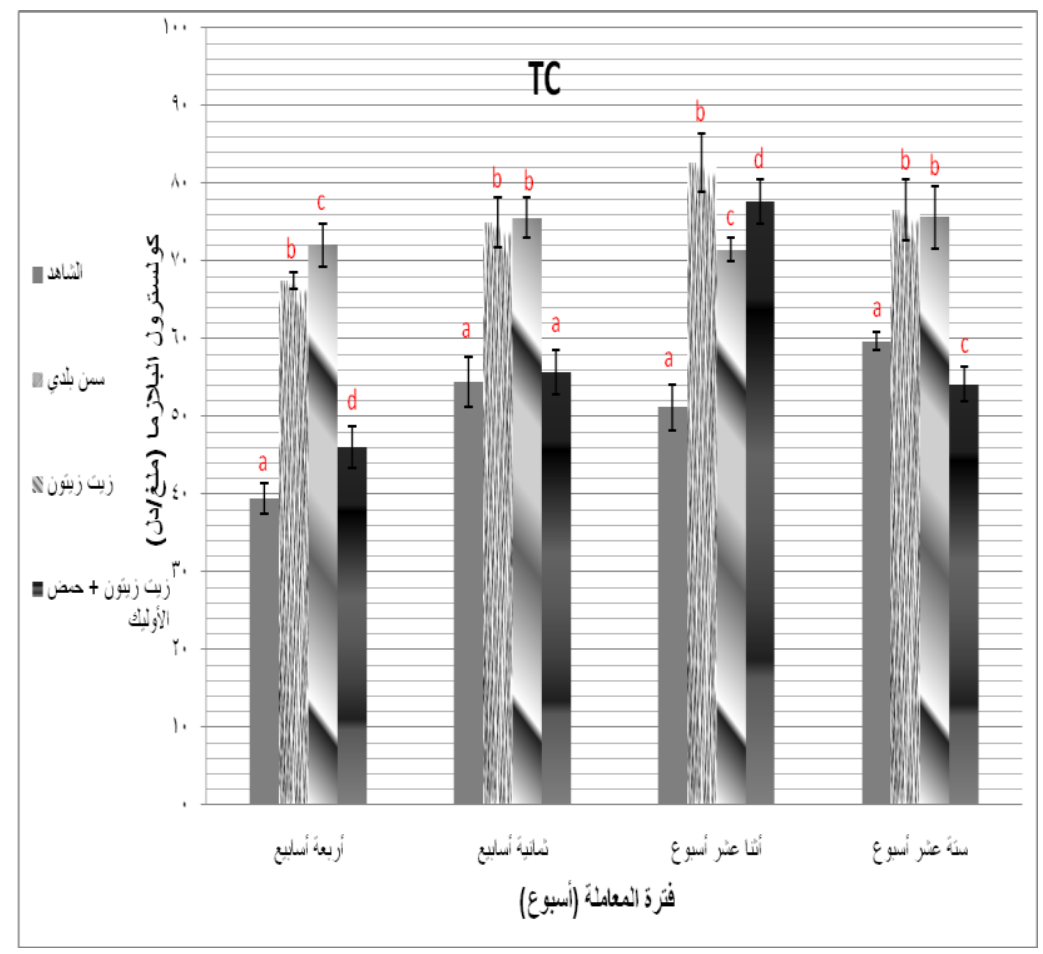

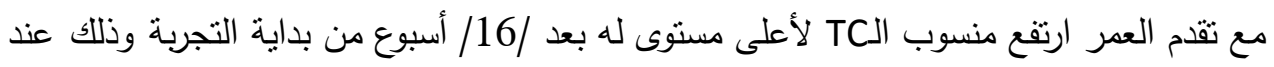

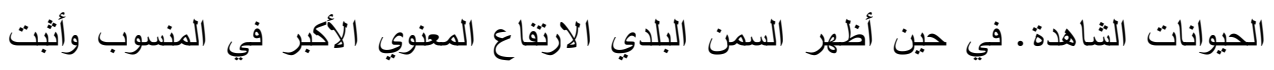

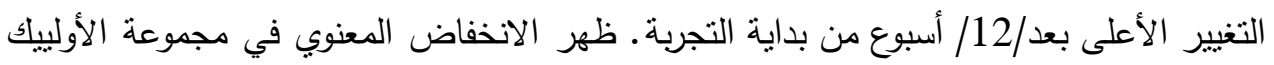

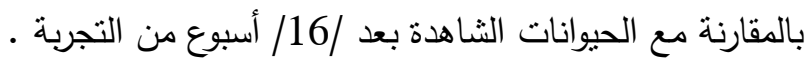


الثكل رقم (2): تركيز الكولسترول -HDL مقدّراً بالملغ/دل في بلازما الدم عند الهمستر في كل من مجموعات (الثناهد والسمن البلدي وزيت الزيتون وزيت الزيتون المضاف له حمض الأولييك

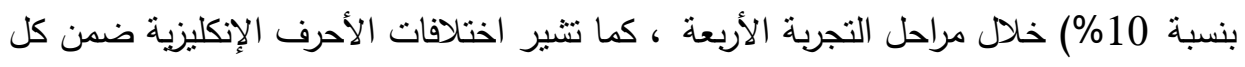
مرحلة تغذية إلى وجود الفروق المعنوية، حيث أنّ ( $0.05>p$ ) ) ، (n=8).

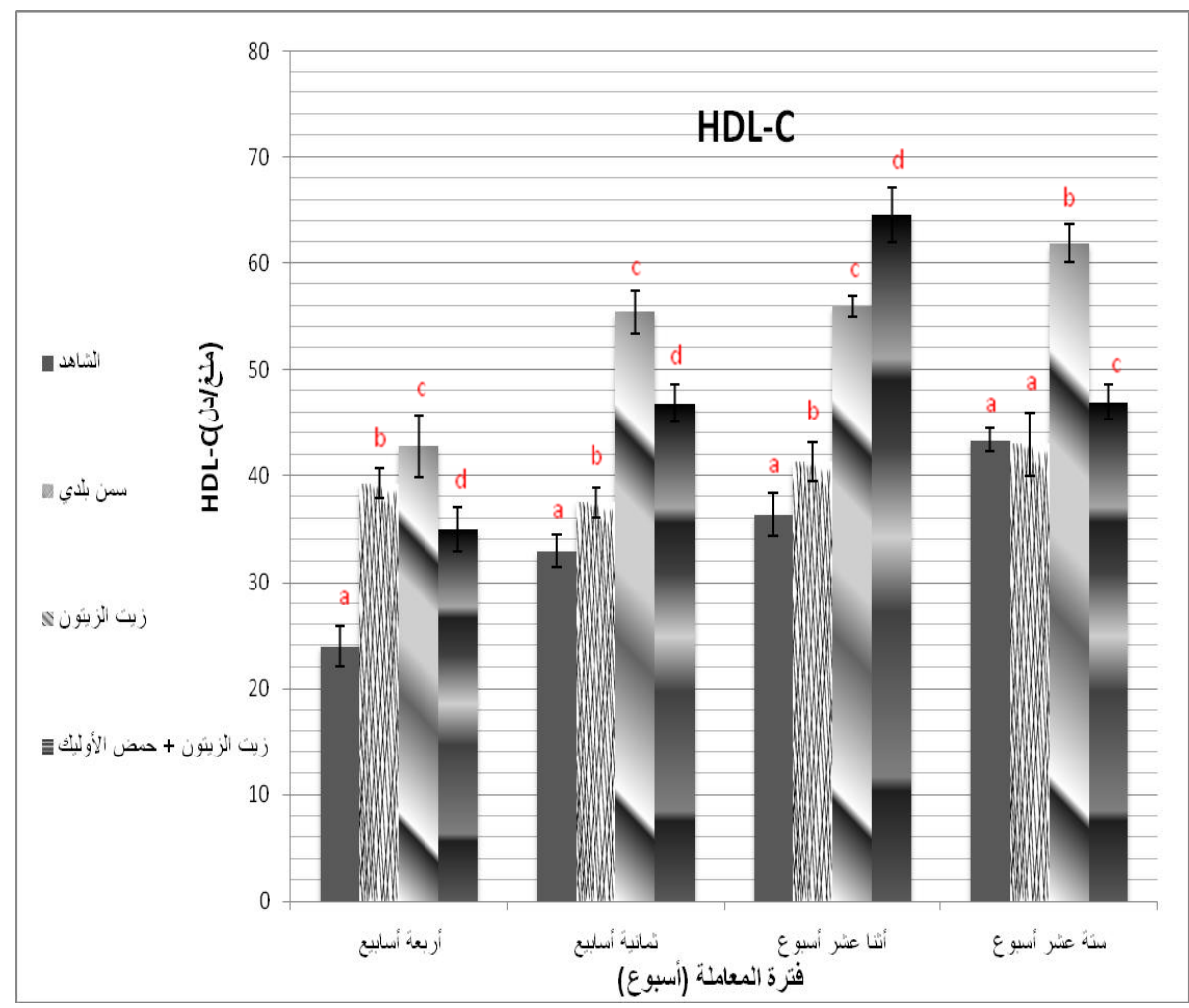

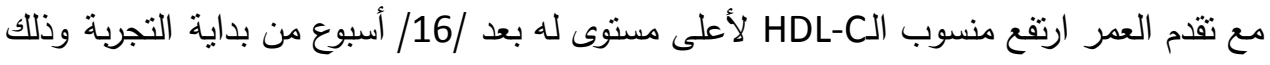

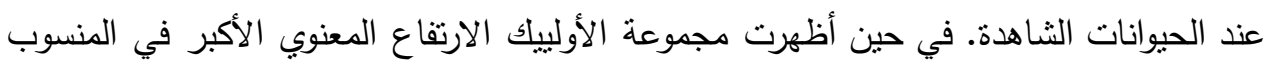
وأثبت التغيير الأعلى بعد/12/ أسبوع من بداية التجربة. 
الثكل رقم ( 3): يمثل النسبة HDL-C/TC في بلازما الدم عند الهمستر في كل من مجموعات

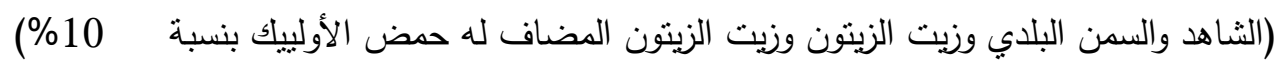

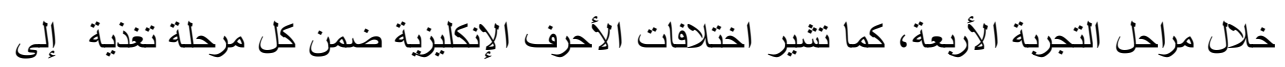

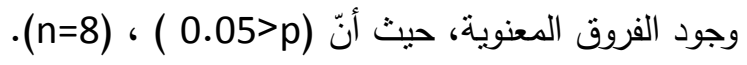

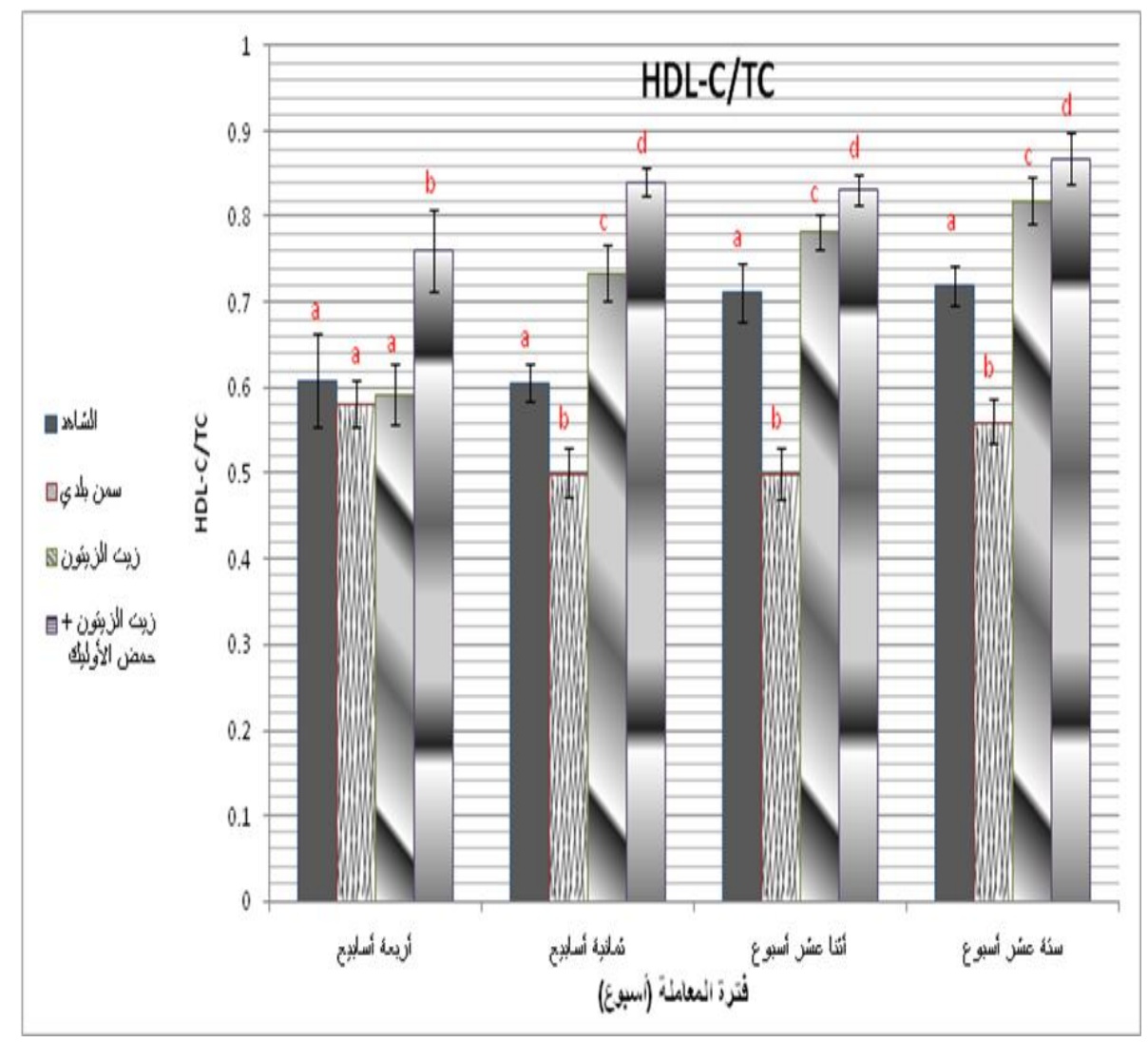

أظهرت مجموعة الأولييك ارتفاعاً معنويًاً في النسبة HDL-C/TC بالمقارنة مع كل المجموعات وفي كل مراحل التجربة وأثثبت التغيير الأعلى بعد/16/ أسبوع من بداية التجربة. في حين التئي

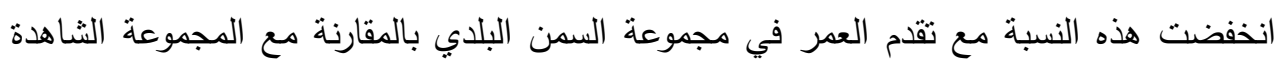
بعد /8/ أسبوع ولوحظ الانخفاض المعنوي الأكبر بعد /12/ أسبوع من المعاملة. 


\section{DISCUSSION

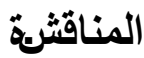

صمّمت هذه الدراسة للنقصتي عن تأثير أهم الدهون المستهلكة (السمن البلاي وزيت

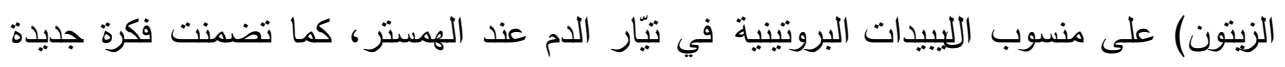

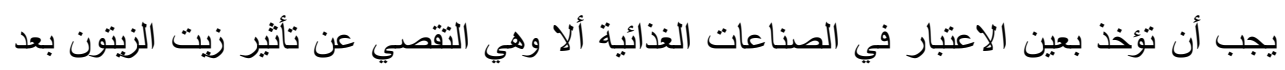

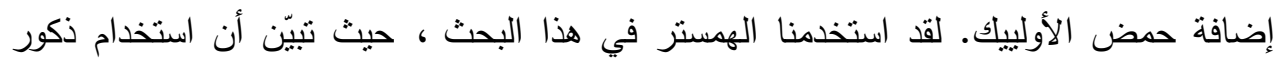

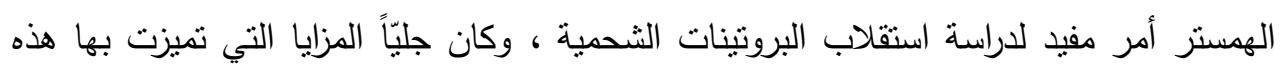

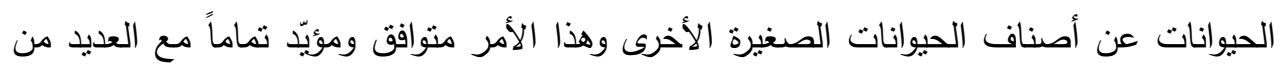

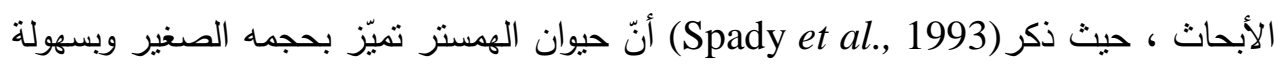

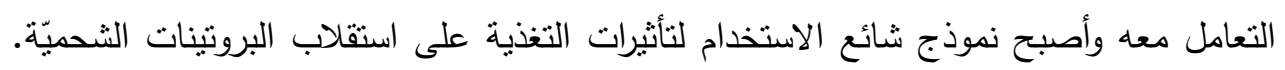

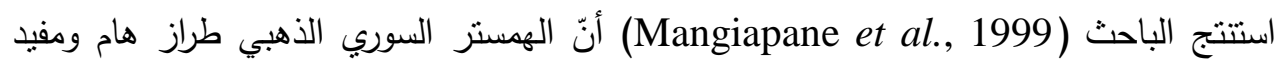
لإستقلاب البروتينات الثحمية ودراسة تطور وانحسار التصلب العصيدي عند الإنسان، كما أنّ النّات

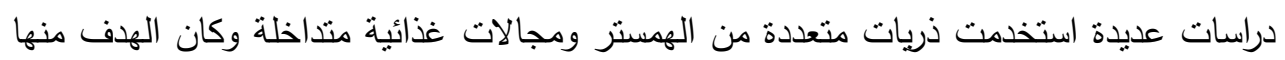

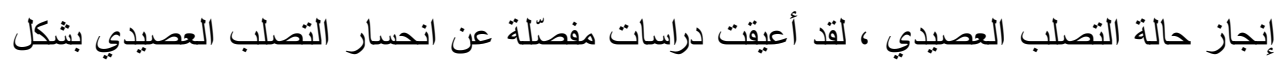

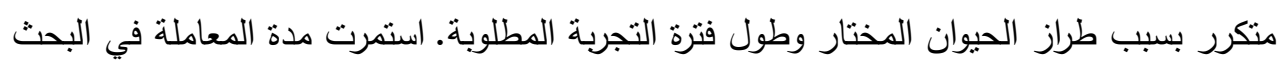

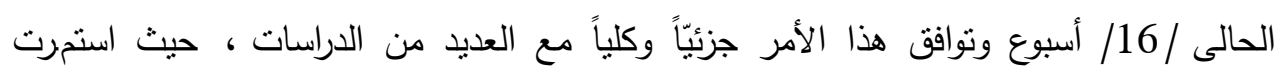

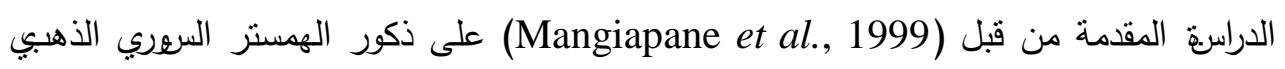
لمدة /4/ أسابيع ، في بحث آخر من قبل (Nicolosi et al., 2004) باستخدام ذكور الهمستر

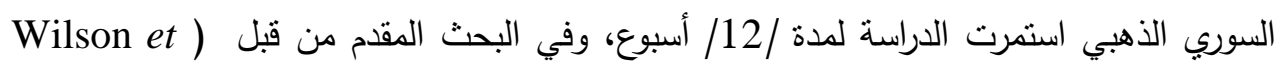

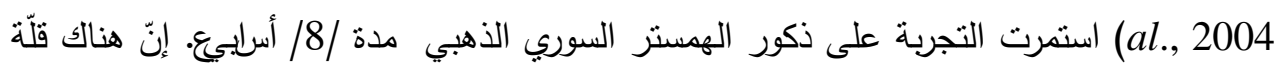

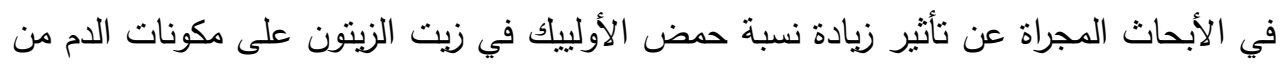

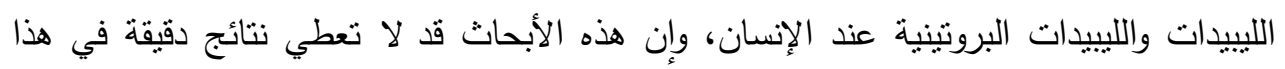

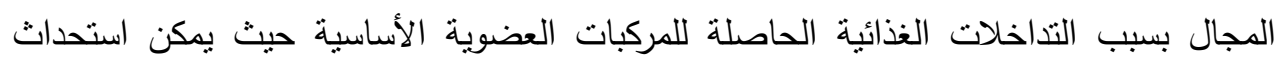

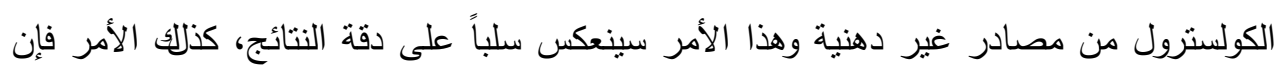

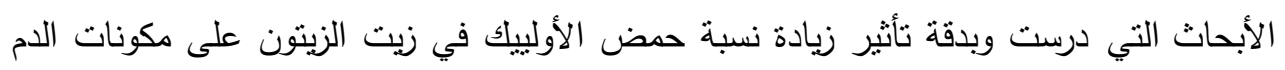

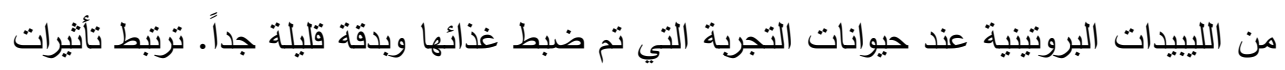

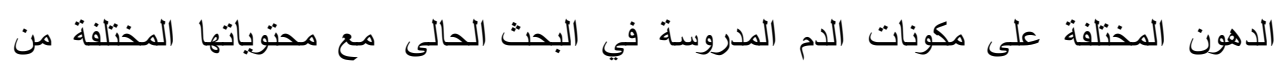


الأحماض الدسمة المشبعة والغير مشبعة ، حيث بات معروفاً أنّ التغذية على الأحماض الدسمة

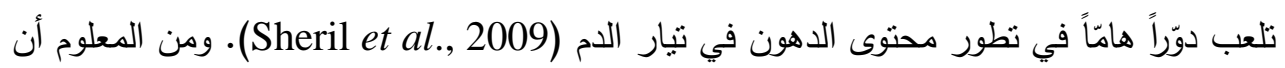

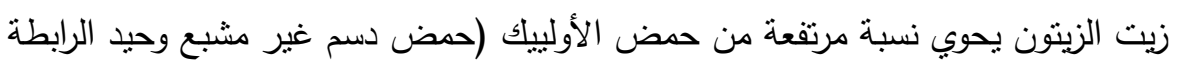

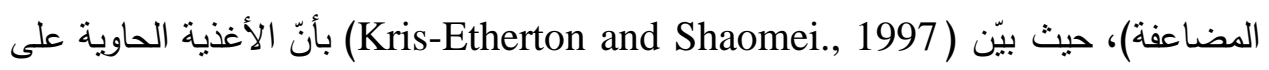
الأحماض الدسمة غير المشبعة وحيدة الرابطة المضاعفة كحضض الأوليك لها تأثير مخفّض

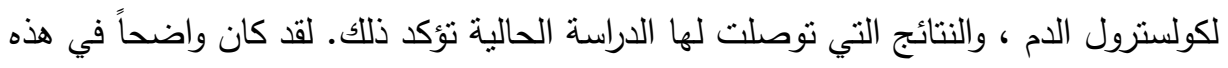
التجربة التأثير الإيجابي لا ل MUFA (أحماض دسمة غير مشبعة وحيدة الرابطة المضاعفة)

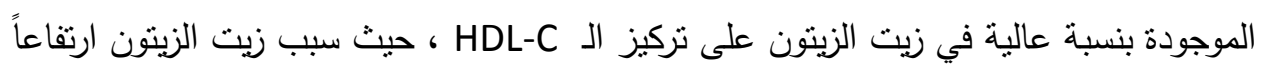

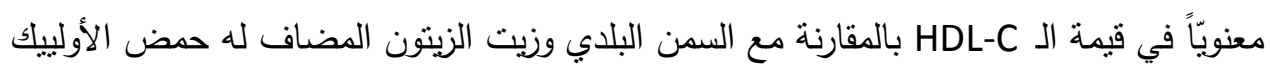

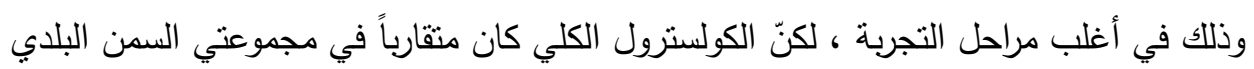

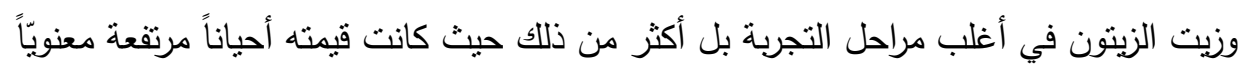

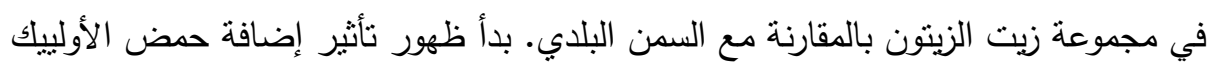

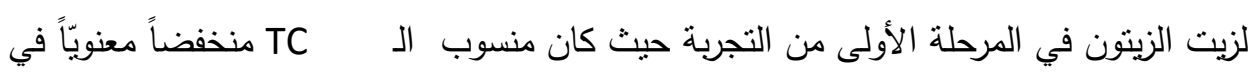

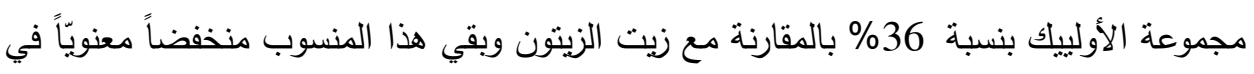

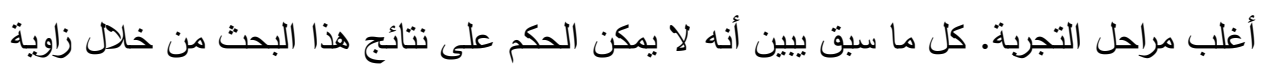

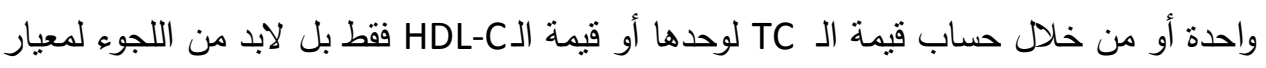
آخر يحسم النتائج بدقة ، ومن هنا تأتي أهيّة حساب النسبة ل معنويًاً في مجموعة الأولييك بالمقارنة مع زيت الزيتون والسمن البلدي وكذللك الثناهد في كل التل

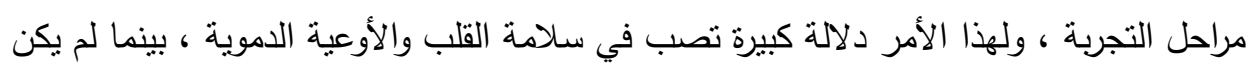

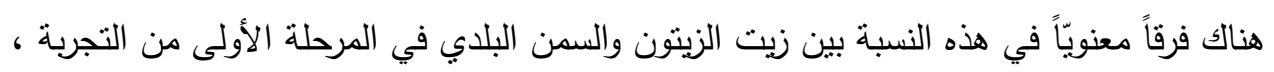

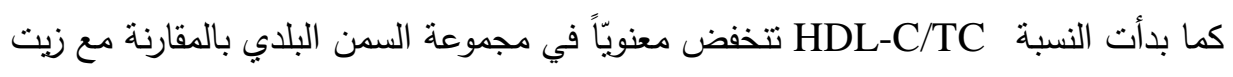

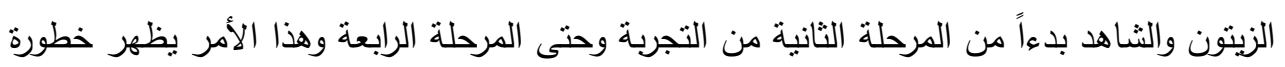

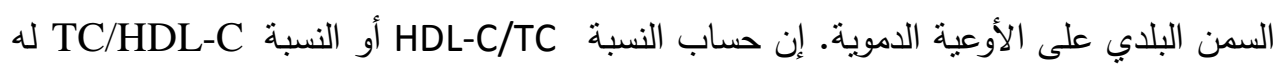

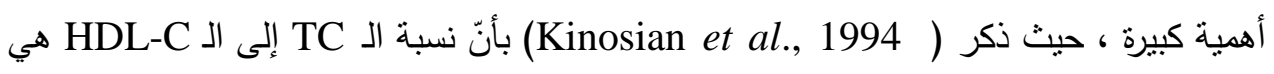

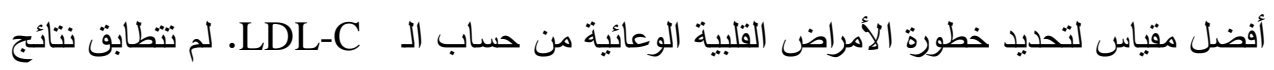
بحثا هذا مع ما جاء به ( Wilson et al., 2004 من حيث منسوب الـ HDL-C عند الهمستر

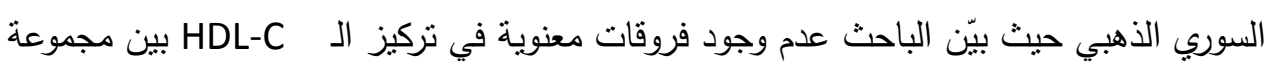

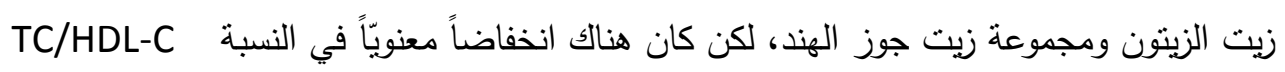


مقداره 23\% في مجموعة زيت الزيتون بالمقارنة مع مجموعة زيت جوز الهند ، حيث تطابقت نتائج دراستتا مع هذه الحقيقة الأخيرة التي بيّنت ضرورة وأهمية حساب النسبة

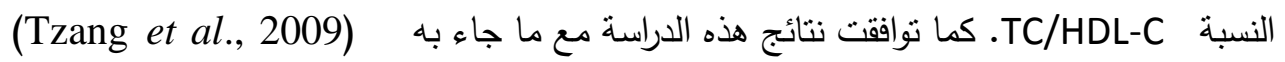
حيث استتنج الأخير وشركاه ارتفاعاً معنوياً في كولسترول الدم (TC) في كل من مجموعني الزبدة

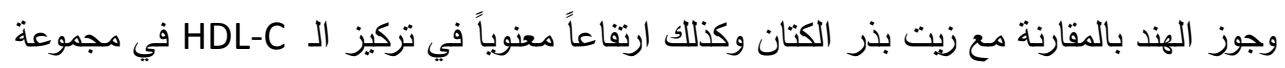

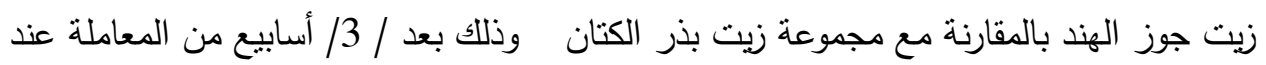

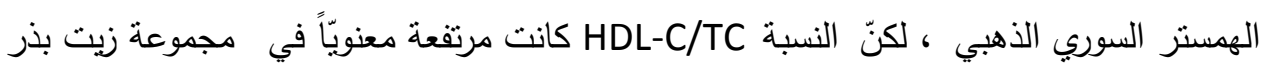

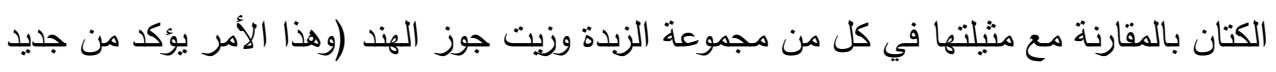
ضرورة حساب النسبة HDL-C/TC في حسم نتائج أي دراسة تتعلق بالكولسترول الكلي وأقسامه) . إنّ دراسات عديدة بيّنت أنّ القوت الدهني المحتوي على أحماض دسمة مشبعة ذات دات السلسلة

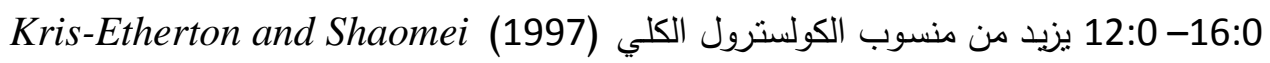

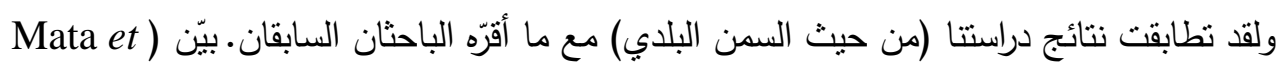
(al., 1992

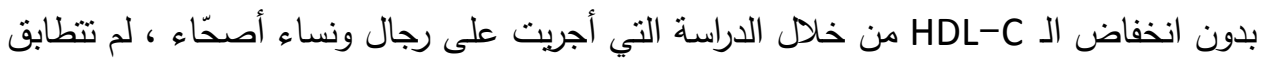

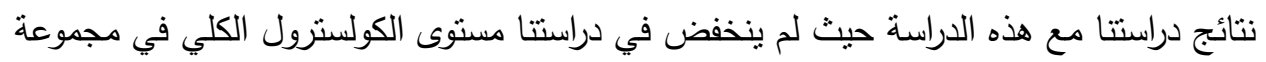

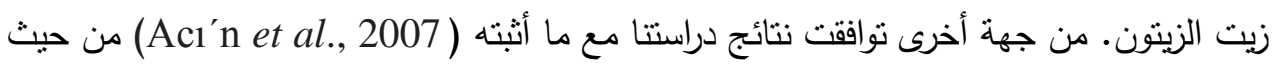
أنّ العليقة المضاف لها زيت الزيتون المثالي بنسبة 10\% أحدثت زيادة معنوية في الكولسترول الكلي، وتخالفت معها من نقطة الزيادة الغير معنوية في منسوب الـ

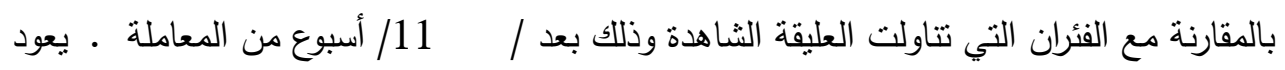
الارتفاع المعنوي الواضح في تركيز الـTC في بلازما دم الهمستر المعامل بالسمن البلدي بالمقاركارنة

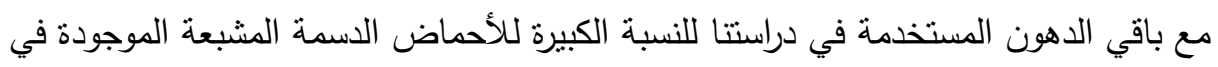

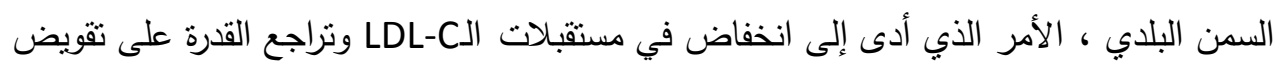

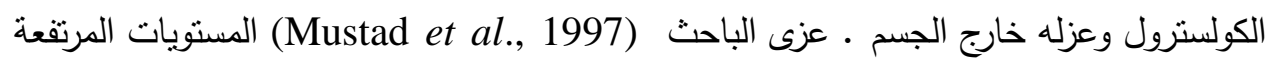
لتركيز الـLDL-C في بلازما دم الجرذان المغذاة على عليقة فرط الكولسترول إلى الخسارة

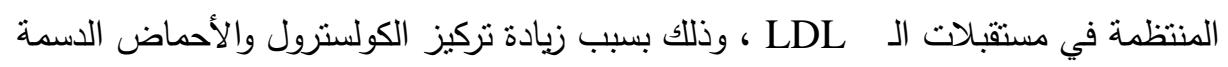

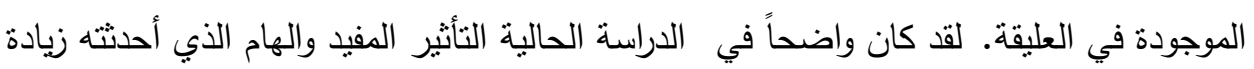

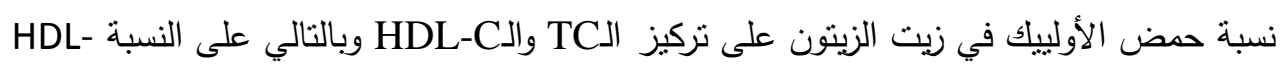
C/TC وذلك بالمقارنة مع زيت الزيتون العادي وظهر التأثير الأعظمي والهام في نهاية التجربة 
عندما انخفض الـ TC معنويًاً في مجموعة الأولييك بالمقارنة مع المجموعة الثاهدة وهذا الأمر له

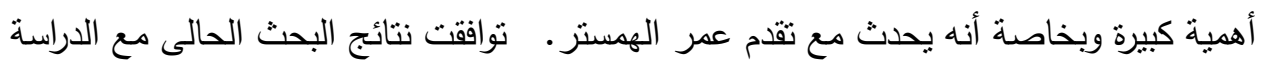
التي أجراها (Perona et al., 2003) والذي أقزّ بانخفاض الكولسترول الكلي عند الإنسان بعد فئس

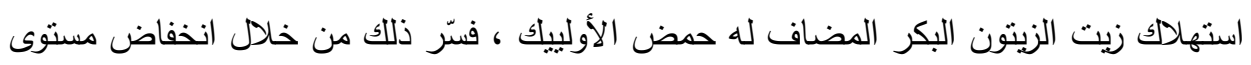

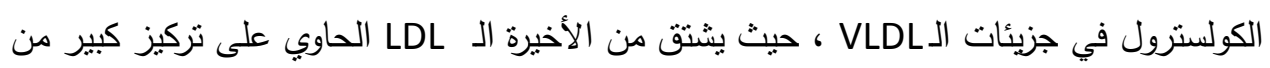

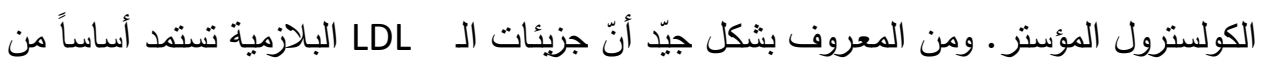

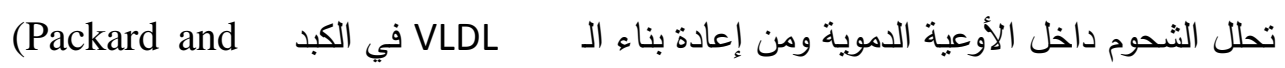
LDL حيث أثنارت براهين عديدة إلى أنّ التركيب والخواص الإستقلابية لا Shepherd 1998) مرتبطة بشكل خالص بجوهر سابقتها الـ GLDL

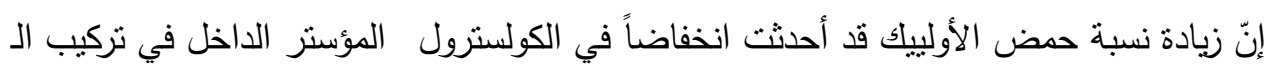

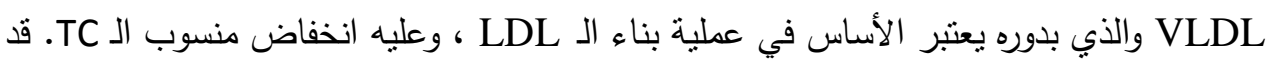

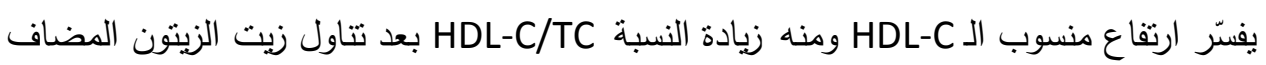

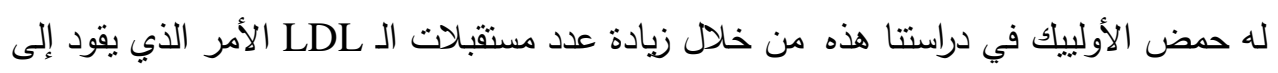

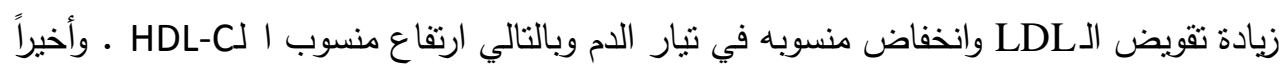

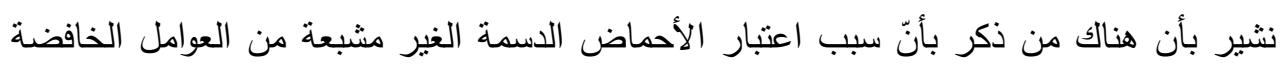

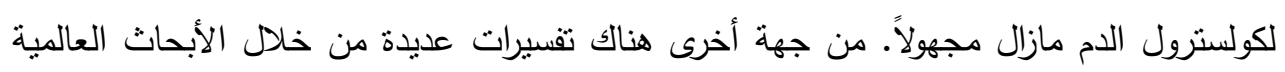

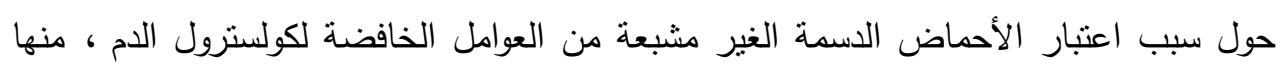

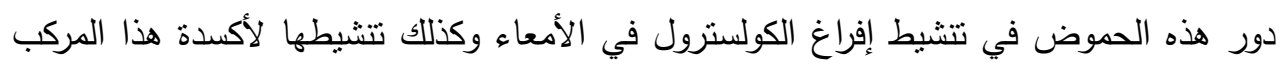

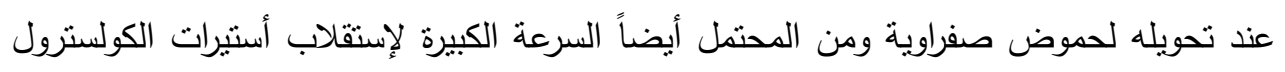
من قبل الكبد وبعض النسج الأخرى وهذا الأمر يعزز عمليتي دوران وإفراغ هذه الأستيرات.

\section{CONCLUSIONS}

\section{الاستتناجات}

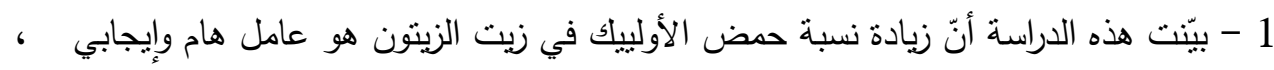

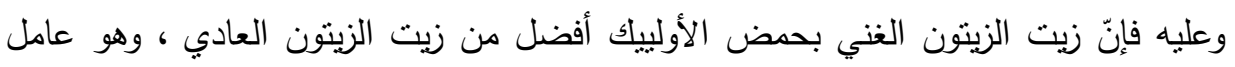


أساسي في زيادة النسبة HDL-C/TC وبالنالي الوقاية من التصلب العصيدي والأمراض القلبية ذات المنشأ الغذائي.

2 - أحدث السمن البلدي تأثيراً خطيراً على مكونات الدم من الليبيدات البروتينية وسبب انخفاضاً

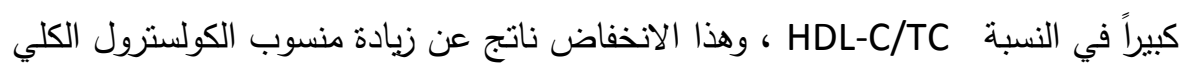

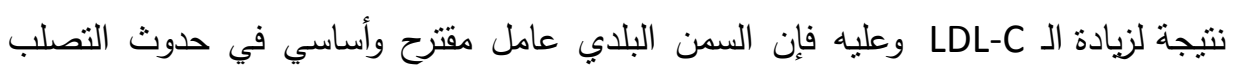
العصيدي وتضيق الثرايين والسكتات القلبية.

3 - بيّنت هذه الدراسة أهمية عدم الاكتفاء بقياس منسوب الكولسترول الكلي فقط لتقدير سلامة ولئه

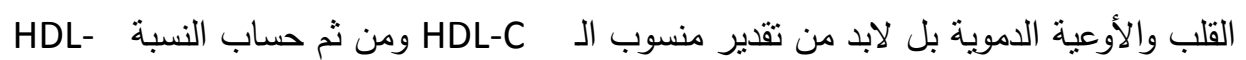
$\mathrm{C} / \mathrm{TC}$ 4 - أظهر الهمستر تحمّلاً كبيراً لجرعات الدهون المتتاولة لفترات طويلة ولذلك فهو طراز مفيد وهام كحيوان تجربة في اختبارات التغذية واستقلاب البروتينات الثحمية.

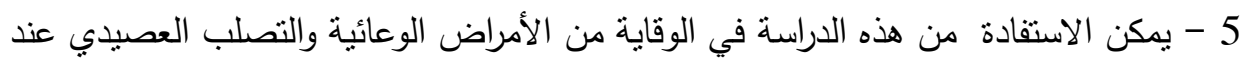

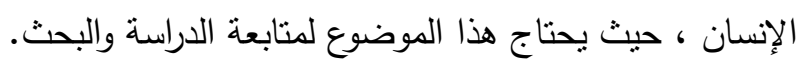

\section{REFERENCES}
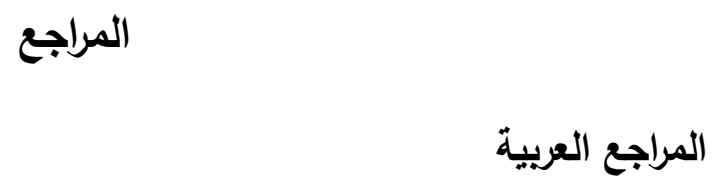

1 - العواد ، عواد (2005) : الكيمياء الحيوية - منشورات جامعة البعث - كلية الطب البيطري.

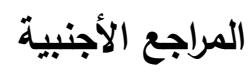

Acl'n, S.; Navarro, M.A.; Perona, J.S.; Mainar, J.M.; Surra, J.C.; Mario A.; Guzma'n, R.C.; Arnal, C.; Orman, I.; Segovia, J.C.O. and Ruiz-Gutierrez, V. (2007): Olive oil preparation determines the atherosclerotic protection in apolipoprotein $\mathrm{E}$ knockout mice Journal of Nutritional Biochemistry, 18: 418- 424.

Allain, C.C.; Poon, L.S. and Chen, C.S.G., et al. (1974): Enzymatic determination of total serum cholesterol. Clin Chem, 20: 470-5.

Bennett, A.J.; Billett, M.A.; Salter, A.M.; Mangiapane, E.H.; Bruce, J.S.; Anderton, K.L.; Marenah, C.B.; Lawson, N. and White, D.A. (1995): Modulation of hepatic lipoprotein B, 3-hydroxy-3methyl-glutaryl- CoA reductase and low density lipoprotein 
receptor mRNA and plasma lipoprotein concentrations by defined dietary fats. Biochemical Journal, 311: 167-173.

Carleton, R.A.; Dwyer, J.; Finberg, L.; Goodman, D.S.; Grundy, S.M.; Havas, S.; Hunter, G.T.; Kritchevsky, D.; Laver, R.M.; Luepker, R.V.; Ramirez, A.G.; Horn, L.V.; Stason, W.B. and Stokes, J. (1991): Report on the expert panel on population strategies for blood cholesterol reduction. A statement from the National Cholesterol Education Program, National Heart, Lung, and Blood Institute, National Institutes of Health. Circulation, 83: 2154-2232.

El-Beshbishy, H.A.; Singab, A.N.B.; Sinkkonen, J. and Pihlaja, K. (2006): Hypolipidemic and antioxidant effects of Morus alba $L$. (Egyptian mulberry) root bark fractions supplementation in cholesterol-fed rats. Life Sciences, 78: 2724-2733.

Guerin, M.; Lassel, T.S.; Le Goff, W.; Farnier, M. and Chapman, M.J. (2000): Action of atorvastatin in combined hyperlipidemia: preferential reduction of cholesteryl ester transfer from HDL to VLDL1 particles. Arterioscler Thromb Vasc Biol, 20: 189.

Harkness, J.E. and Wagner, E. (1995): The biology and medicine of rabbits and rodents. 4 th ed. Baltimore.

Jain, D.; Ebine, N.; Jia, X.; Kassis, A.; Marinangeli, C.; Fortin, M.; Beech, R.; Kevin, B.; Robert, H.; Moreau, A.; Kubow, S. and Peter, J.H. Jones (2008): Corn fiber oil and sitostanol decrease cholesterol absorption independently of intestinal sterol transporters in hamsters. Journal of Nutritional Biochemistry, 19: 229-236.

Kinosian, B.; Glick, H. and Garland, G. (1994): Cholesterol and coronary heart disease: Predicting risks by levels and ratios. Ann Intern Med , 121: 641-647.

Kris-Etherton, P.M. and Shaomei, Y. (1997): Individual fatty acid effects on plasma lipid and lipoproteins: human studies. Am J Clin Nutr, 65: 1628-44.

Mangiapane, E.H.; Martina, A.; McAteer, G.; Benson, M. and David, A. (1999): Modulation of the regression of atherosclerosis in the hamster by dietary lipids: comparison of coconut oil and olive oil, British Journal of Nutrition, 82: 401-409.

Martinez-Flores, E.; Chang, Y.K.; Martinez-Bustos, F. and Sgarbieri, V. (2004): Effect of high fiber products on blood lipids and lipoproteins in hamsters. Nutrition Research, 24: 85-93.

Mata, P.; Alvarez-Sala, L.; Rubio, M.; Nuño, J. and De Oya, M. (1992):

Effects of long-term monounsaturated- vs polyunsaturated- 
enriched diets on lipoproteins in healthy men and women. Am J Clin Nutr, 55: 846-50.

Mustad, V.A.; Etherton, T.D.; Cooper, A.D.; Mastro, A.M.; Pearson, T.A.; Jonnalagadda, S.S. and Kris-Etherton, P.M. (1997): Reducing saturated fat intake is associated with increased levels of LDLreceptors on mononuclear cells in healthy men and women. Journal of Lipid Research, 38: 459- 468.

Nicolosi, R.J.; Woolfrey, B.; Wilson, T.A.; Scollin, P.; Handelman, G. and Fisher, R. (2004): Decreased aortic early atherosclerosis and associated risk factors in hypercholesterolemic hamsters fed a high- or mid-oleic acid oil compared to a high-linoleic acid oil. Journal of Nutritional Biochemistry, 15: 540-547.

Nielsen, L.B.; Perleth-Espensen, Bbrge G.; Foged, N.E.; Kjeldsen, K. and Stender, S. (1995): Replacement of dietary saturated fat with monounsaturated fat: effect on atherogenesis in cholesterol-fed rabbits clamped at the same plasma cholesterol level. British Journal of Nutrition, 14: 509-521.

Packard, C.J. and Shepherd, J. (1998): Regulation of VLDL production in man: implications for coronary heart disease (CHD). In: Jacotot B, Mathe D, Fruchart J-C, eds. Atherosclerosis, Vol XI. Singapore: Elsevier Science, 789.

Perona, J.S.; Can izares, J.; Montero, E.; Sa'nchez-Domı, J.M. and RuizGutierrez, V. (2003): Plasma lipid modifications in elderly people after administration of two virgin olive oils of the same variety with different triacylglycerol composition, 89: 819.

Rosamond, W.; Flegal, K.; Furie, K.; Go, A.; Greenlund, K. and Haase, N. et al. (2008): Heart disease and stroke statistics. (2008): update: A report from the American Heart Association Statistics Committee and Stroke Statistics Subcommittee. Circulation, 117: 25-146.

Salter, A.M.; Mangiapane, E.H.; Bennett, A.J.; Bruce, J.S.; Bilett, M.A.; Anderton, K.L.; Marenah, C.B.; Lawson, N. and White, D.A. (1998): The effect of different dietary fatty acids on lipoprotein metabolism: concentration-dependent effects of diets enriched in oleic, myristic, palmitic and stearic acids. British Journal of Nutrition, 79: 195-202.

Sessions, V.A. and Salter, A.M. (1994): The effects of different dietary fats and cholesterol on serum lipoprotein concentrations in hamsters. Biochimica et Biophysica Acta, 1211: 207-214.

Sheril, A.; Jeyakumar, S.M.; Jayashree, T.; Giridharan, N.V. and Vajreswari, A. (2009): Impact of feeding polyunsaturated fatty 
acids on cholesterol metabolism of dyslipidemic obese rats of WNIN/GR-Ob strain. Atherosclerosis, 204: 136-140.

Smith, J.R.; Jackson, S.C.; Pearson, R.; Fuster, T.A.; Yusuf, V.; Faergeman, S.; Wood, O.; Alderman, D.A.; Horgan, M.; Home, J.; Hunn, P. and Grundy, M.S.M. (2004): Principles for national and regional guidelines on cardiovascular disease prevention: a scientific statement from the World Heart and Stroke Forum. Circulation, 109: 3112-3121.

Spady, D.K.; Woollett, L.A. and Dietschy, J.M. (1993): Regulation of plasma LDL-cholesterol by dietary cholesterol and fatty acids. Annual Reviews of Nutrition, 13: 355-381.

Tzang; Bor-Show.; Yang; Shun-Fa.; Fu, Shih-Guei.; Yang, Hui-Chun.; Sun, Hai-Lun. and Chen, Yi-Chen. (2009): Effects of dietary flaxseed oil on cholesterol metabolism of hamsters. Food Chemistry, 114: 1450-1455.

Weingand, K.W. and Daggy, B.P. (1990): Quantitation of high- density lipoprotein cholesterol in plasma from hamsters by differential precipitation. Clin Chem, 36: 575.

White, D.A.; Bennett, A.J.; Billett, M.A. and Salter, A.M. (1997): Genetic determinants of plasma lipoprotein levels and their dietary response. Prostaglandins, Leukotrienes and Essential Fatty Acids, 57: 455-462.

Wilson, T.A.; Kritchevsky, D.; Kotyla, T. and Nicolosi, R.J. (2006): Structured triglycerides containing caprylic (8:0) and oleic (18:1) fatty acids reduce blood cholesterol concentrations and aortic cholesterol accumulation in hamsters. Biochimica et Biophysica Acta, 1761: 345-349.

Wilson, T.A.; Nicolosi, R.J.; Yoganathan, G.H.S.; Kotyla, T.; Orthoefer, F. and Binford, P. (2004): Comparative effects of emu and olive oil on aortic early atherosclerosis and associated risk factors in hypercholesterolemic hamsters. Nutrition Research, 24:395-406. 
Assiut Vet. Med. J. Vol. 56 No. 127 October 2010

$-21-$ 